\title{
Does nociception monitor-guided anesthesia affect opioid consumption? A systematic review of randomized controlled trials
}

\author{
Fleur S. Meijer ${ }^{1} \cdot$ Marieke Niesters $^{1} \cdot$ Monique van Velzen $^{1} \cdot$ Chris H. Martini ${ }^{1} \cdot$ Erik Olofsen $^{1} \cdot$ Ruth Edry $^{2}$. \\ Daniel I. Sessler ${ }^{3} \cdot$ Eveline L. A. van Dorp $^{1} \cdot$ Albert Dahan $^{1} \cdot$ Martijn Boon $^{1}$ (i)
}

Received: 25 February 2019 / Accepted: 16 July 2019 / Published online: 20 July 2019

(c) The Author(s) 2019

\begin{abstract}
Monitors that estimate nociception during anesthesia may be used to guide opioid and other analgesics administration to optimize anesthesia care and possibly outcome. We reviewed the literature to evaluate current evidence of the effect of nociception-guided management over standard anesthesia practice during surgery. A systematic review of the literature on the effect of nociception monitoring on anesthesia practice was conducted. Reports were eligible if they compared nociception-guided anesthesia to standard practice during surgery. Primary endpoint of this review is intraoperative opioid consumption. Secondary endpoints included hemodynamic control, postoperative pain and pain treatment. We identified 12 randomized controlled trials that compared one of five different nociception monitoring techniques to standard anesthesia care. Most studies were single center studies of small sample size. Six studies reported intraoperative opioid consumption as primary outcome. There was considerable variability with respect to surgical procedure and anesthesia technique. For nociception monitors that were investigated by more than one study, analysis of the pooled data was performed. The surgical plethysmographic index was the only monitor for which an intra operative opioid sparing effect was found. For the other monitors, either no effect was detected, or pooled analysis could not be performed due to paucity of study data. On secondary outcomes, no consistent effect of nociception-guided anesthesia could be established. Although some nociception monitors show promising results, no definitive conclusions regarding the effect of nociception monitoring on intraoperative opioid consumption or other anesthesia related outcome can be drawn.
\end{abstract}

Clinical trial number PROSPERO ID 102913.

Keywords Nociception monitoring · Opioid consumption · Systematic review

Electronic supplementary material The online version of this article (https://doi.org/10.1007/s10877-019-00362-4) contains supplementary material, which is available to authorized users.

Martijn Boon

m.boon@lumc.nl

Fleur S. Meijer

f.s.meijer@lumc.nl

Marieke Niesters

m.niesters@lumc.nl

Monique van Velzen

m.van_velzen@lumc.nl

Chris H. Martini

c.h.martini@lumc.nl

Erik Olofsen

e.olofsen@lumc.nl

Ruth Edry

ruedry@gmail.com
Daniel I. Sessler

ds@or.org

Eveline L. A. van Dorp

e.1.a.van_dorp@lumc.nl

Albert Dahan

a.dahan@lumc.nl

1 Department of Anesthesiology, Leiden University Medical Center, Albinusdreef 2 (Postal Zone H5-Q), 2333 ZA Leiden, The Netherlands

2 Department of Anesthesiology, Rambam Medical Centre, The Ruth and Bruce Rappaport Faculty of Medicine, Technion Institute of Technology, Haifa, Israel

3 Department of Outcomes Research, Anesthesiology Institute, Cleveland Clinic, Cleveland, OH, USA 


\section{Introduction}

General anesthesia is intended to produce a state of unconsciousness combined with suppression of nociception, allowing the patient to undergo invasive surgical procedures without undue harm or awareness. Nociception is defined by the International Association for the Study of Pain as the neural process of encoding noxious stimuli, causing autonomic and/or behavioral responses such as elevation of blood pressure or motor withdrawal reflexes; noxious stimuli are actually or potentially tissue damaging events that occur during surgery [1]. Nociception is generally suppressed by administration of potent opioid analgesics.

Clinicians usually estimate nociception by evaluating hemodynamic responses, along with lacrimation, sweating, increase in pupil diameter or movement. Recently, nociception monitors have been introduced to track nociception during anesthesia and guide administration of analgesics, usually opioids. Since inadequate opioid administration is associated with unwanted hemodynamic responses (e.g. hypertension and hypotension), reliable nociception monitors may help optimize anesthetic management. Evidence from preclinical validation studies show that these monitors distinguish noxious and non-noxious events far better than hemodynamic responses [2-6].

Various nociception monitors are already available, and others are being developed. All use algorithms to assess various physiological variables and they produce numerical indexes that give an estimation of the nociception-antinociception balance (see Table 1). The value of nociception monitors is increasingly assessed in clinical settings, where they are compared to standard anesthesia care. We intended to evaluate current evidence of the effect of nociception-guided management on intraoperative opioid consumption and other anesthesia related outcomes. To this end, a systematic search was conducted to identify reports that assessed intraoperative nociception monitoring versus routine anesthetic management on intraoperative opioid consumption. If possible, analysis of pooled data was performed to synthesize current evidence.

\section{Materials and methods}

Our goal was to determine whether the use of a monitor or algorithm that estimates patients' nociceptive state during general anesthesia alters anesthesia management with respect to the administration of opioids. Secondary aims were to evaluate the effect of nociception monitoring on hemodynamic parameters, time related variables (e.g. time to extubation) and postoperative pain score and opioid consumption. We searched for trials in which the primary endpoint was the amount of opioid analgesic medication given during anesthesia. All available nociception monitors were considered, along with indices derived from the electroencephalogram or evoked potentials. The systematic review was conducted in accordance with the PRISMA statements. A planned meta-analysis was registered at PROSPERO (www.crd.york.ac.uk/prospero) under Identifier 102913. Since there was so much heterogeneity among the included studies, a valid meta-analysis could not be conducted. Instead, pooled data were analyzed exclusively within unique monitors, when available.

\subsection{Identification of relevant studies}

On June 30, 2018 we searched the PubMed electronic database from inception for studies on nociception monitoring. The search strategy is given in Supplemental Document 1; no language or date restrictions were applied. To reduce the risk of missing relevant studies, we checked relevant review papers and a previous meta-analysis [7]. The title
Table 1 Individual nociception monitor characteristics

\begin{tabular}{lllll}
\hline Monitor & Manufacturer & Autonomic input variables & Index & Optimal range/cut off \\
\hline SPI & GE Healthcare & $\begin{array}{l}\text { Pulse beat interval } \\
\text { Pulse wave amplitude }\end{array}$ & $0-100$ & $20-50$ \\
ANI & $\begin{array}{c}\text { MDoloris } \\
\text { Medical } \\
\text { Systems }\end{array}$ & Heart rate variability & $0-100$ & 50 \\
NOL & Medasense & $\begin{array}{l}\text { Heart rate and heart rate variability } \\
\text { Pulse wave amplitude }\end{array}$ & $0-100$ & $10-25$ \\
& Skin conductance & & \\
CARDEAN & Alpha-2 & $\begin{array}{l}\text { Beart pressure } \\
\text { Pupil diameter }\end{array}$ & $0-100$ & 60 \\
Algiscan & ID Med & NA & Pupil diameter increase $>30 \%$
\end{tabular}

SPI surgical plethysmographic index, $A N I$ analgesia nociception index, $N O L$ nociception level index, $N A$ not available 
and abstracts of the retrieved studies were next step-wise evaluated for the following three criteria: (1) study in surgical patients, aged 18 years and older, (2) study performed during general anesthesia, and (3) randomized trial of nociception monitor-guided administration of opioids versus standard clinical care in which analgesics were administered solely based on blood pressure and heart rate values. All papers meeting all criteria were read in full. Three reviewers (FM, AD, and RE) independently performed the selection procedure.

\subsection{Data extraction}

The identified reports were searched for the following variables and these were extracted for the review if available: authors, country of origin, year of publication, number of subjects in each treatment group, type of opioid and anesthetic used, opioid consumption during surgery, anesthetic consumption during surgery, duration of anesthesia, hypotension and/or bradycardia events, hypertension and/or tachycardia events, time from end-of-anesthesia or administration of reversal agent until extubation or emergence, duration of stay in the post-anesthesia care unit (PACU), average pain level in the PACU, and opioid consumption in the PACU.

Opioid consumption during surgery was transformed to morphine dose (in $\mathrm{m} \mathrm{kg}^{-1} \mathrm{~h}^{-1}$ ) using the following conversion ratios: $1 \mathrm{mg}$ morphine (intravenous) $=0.5 \mathrm{mg}$ oxycodone $=10 \mu \mathrm{g}$ fentanyl $=1 \mu \mathrm{g}$ sufentanil $=10 \mu \mathrm{g}$ remifentanil $=50 \mu \mathrm{g}$ alfentanil. These conversion rates are arbitrarily based on existing opioid potency data [8].

\subsection{Bias assessment}

Study quality was evaluated with the Cochrane Collaboration's tool for assessing risk of bias in randomized trials [9]. This tool considers six domains of bias: (1) selection bias which includes the presence of random sequence generation and allocation concealment, (2) performance bias which includes the blinding of participants and personnel, (3) detection bias which includes the blinding of outcome assessment, (4) attrition bias, which includes incomplete outcome data, (5) reporting bias which includes selective reporting, and (6) other bias. For each study, the presence of bias in each domain was assessed independently by two reviewers (FM and MN). Discrepancies in judgment were resolved by consensus and, when required a third reviewer was consulted (AD).

\subsection{Data analysis}

Analyses of combined data per unique monitor were conducted using the statistical package $\mathrm{R}$ (version 3.5.0) with the metafor package $[10,11]$. Data were analyzed using random effects models, assuming two sources of variance, within-study error and between-study error. Heterogeneity was by measuring the degree of inconsistency in the studies' results $\left(\mathrm{I}^{2}\right)$.

\section{Results}

\subsection{Study selection}

The flow chart of the PubMed search is shown in Fig. 1, which illustrates retrieval of 741 records. After removal of 728 irrelevant studies, 13 trials were carefully examined and assessed for eligibility. Three papers were removed, one study using the analgesia nociception index (ANI), the other two the surgical plethysmographic index (SPI, Fig. 1) [12-14]. Two of these studies were observational (using a historic control group) or case-control studies [13, 14]; the third study did not report data for the complete duration of the surgical procedure [12]. Finally, two recently published studies were added. The first is a randomized trial from our research group that compared nociception level (NOL)guided analgesia with standard clinical care in patients undergoing major abdominal surgery [15]. The second study is a trial that compared SPI guided anesthesia with standard care during laparoscopic cholecystectomy [16]. Our review process therefore resulted in a total of 12 unique studies eligible for inclusion in the review.

\subsection{Study characteristics}

Characteristics of the 12 included studies are shown in Table 2 and the main findings are summarized in Table 3. All publications were in English. A total of 1045 patients were studied, with 520 receiving an intervention compared to 526 treated according to routine clinical care. Care guided using the SPI was evaluated in six studies [16-21] followed by the ANI in three studies [22-24]. The NOL, pupillometry and the beat-to-beat cardiovascular depth of anesthesia index (CARDEAN 2.0) were evaluated in single studies [15, 25, 26]. Next, a discussion of the available evidence for each separate monitor is presented.

\subsubsection{Surgical plethysmographic index (SPI)}

Six reports were identified that compared SPI guided anesthesia to standard practice [16-21]. Studies were diverse with regard to the maintenance hypnotic (propofol or sevoflurane) and opioids (remifentanil/sufentanil/ fentanyl and oxycodone). The guidance of these agents was however uniform: all maintained a bispectral index or state entropy value between 40 and 60 and steered their SPI values in the intervention group to maintain values 
Fig. 1 Flow diagram of the study selection process following the PubMed search on June 30, 2018

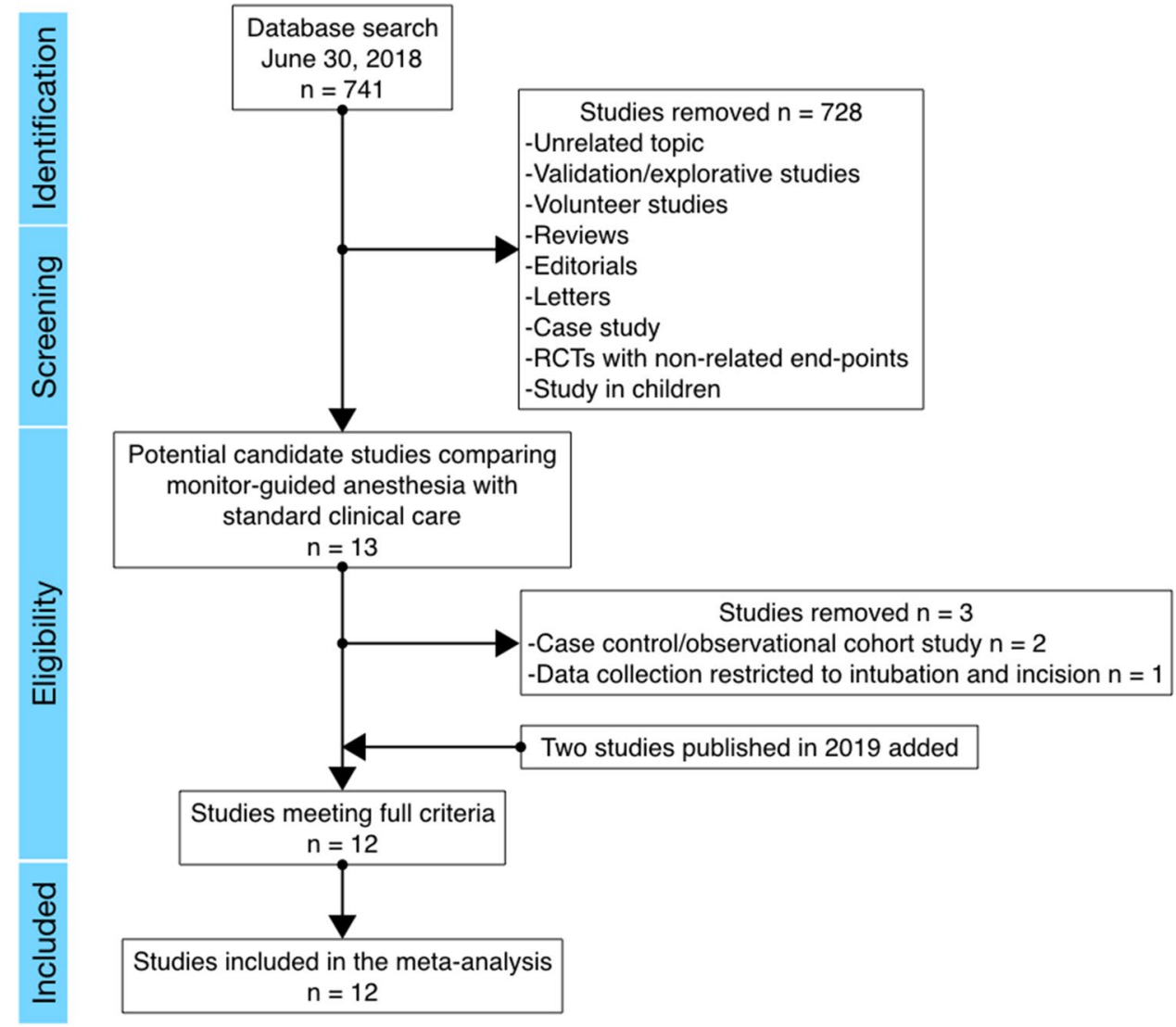

below 50. Bergman et al. and Chen et al. found that SPI monitoring reduced remifentanil consumption during ear-, nose- and throat surgery and orthopedic surgery by $23 \%$ and $25 \%$ respectively $[17,18]$. Similarly, Won et al. found a $30 \%$ reduction in oxycodone consumption in SPI guided patients for thyroid surgery [21]. However, in absolute values, the reduction in oxycodone from that study equaled to only $2.8 \mathrm{mg}$ morphine. In contrast with these studies, Jain et al. found an increased consumption of fentanyl in SPI guided patients during laparoscopic cholecystectomy [16]. This translated to less postoperative pain and a reduced need for postoperative adjuvant analgesia. All other studies failed to find an effect of SPI guidance on postoperative pain or opioid consumption (see Table 2). In general, the effect of SPI guidance on secondary endpoints was limited. No significant differences in mean hemodynamic values were reported, with the exception of Chen et al., who found that the number of episodes with inadequate anesthesia (a composite endpoint that includes hyper/ hypotension and brady/tachycardia) was reduced by $80 \%$ under SPI guidance [18]. Recovery times were identical or at best 4 min faster with SPI guidance. Finally, the studies of Colombo et al. [19] and Gruenewald et al. [20] found no differences on intraoperative opioid consumption or perioperative secondary outcomes at all.
Pooled data analysis shows that the SPI had an overall significant opioid sparing effect: mean difference in morphine equivalents $-0.06 \mathrm{mg} \mathrm{kg}^{-1} \mathrm{~h}^{-1}(95 \% \mathrm{CI}-0.12$ to $-0.00, \mathrm{Z}=-2.0, \mathrm{p}=0.04, \mathrm{I}^{2}=70 \%$ ), or an $8 \%$ reduction in intra operative opioid consumption.

In conclusion, analysis of the pooled data showed that SPI guided management may reduce opioid consumption during surgery, although individual study results varied considerably. Heterogeneity was substantial due to differences in methodology, including type of surgery and choice of opioid and hypnotic agents. Therefore, no definitive conclusions can be drawn.

\subsubsection{Analgesia nociception index (ANI)}

Three reports were identified comparing the ANI to standard of care during lumbar discectomy [24], breast surgery [22] and laparoscopic cholecystectomy [23] (see Table 2). All studies used a volatile hypnotic for maintenance (sevoflurane or desflurane). Fentanyl, remifentanil and morphine were used for analgesia. Dundar et al. provided pre-operative single shot thoracic paravertebral blockade for 44 patients receiving breast surgery under general anesthesia [22]. This study found a significant reduction of intra operative remifentanil consumption in the ANI guided group 


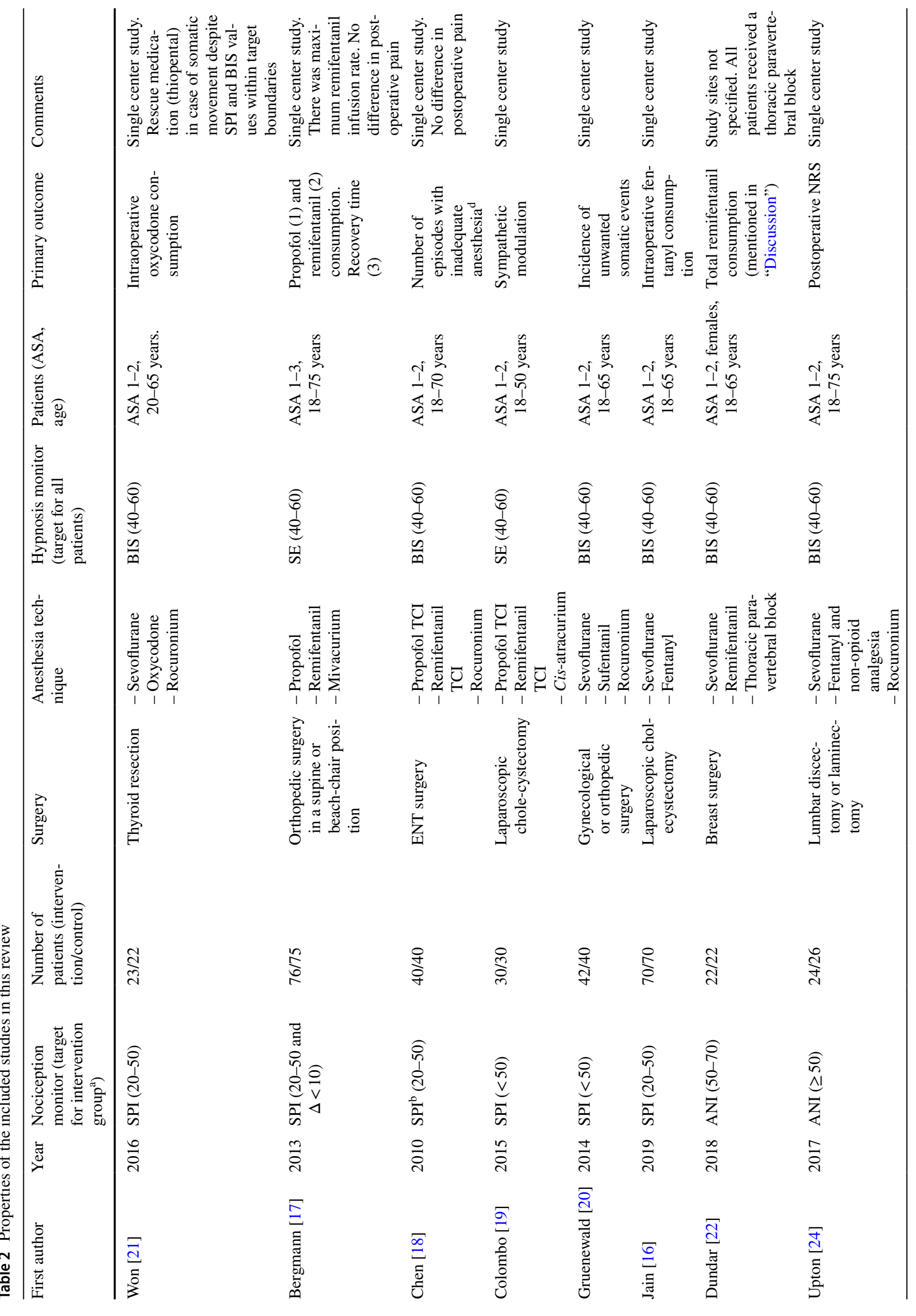




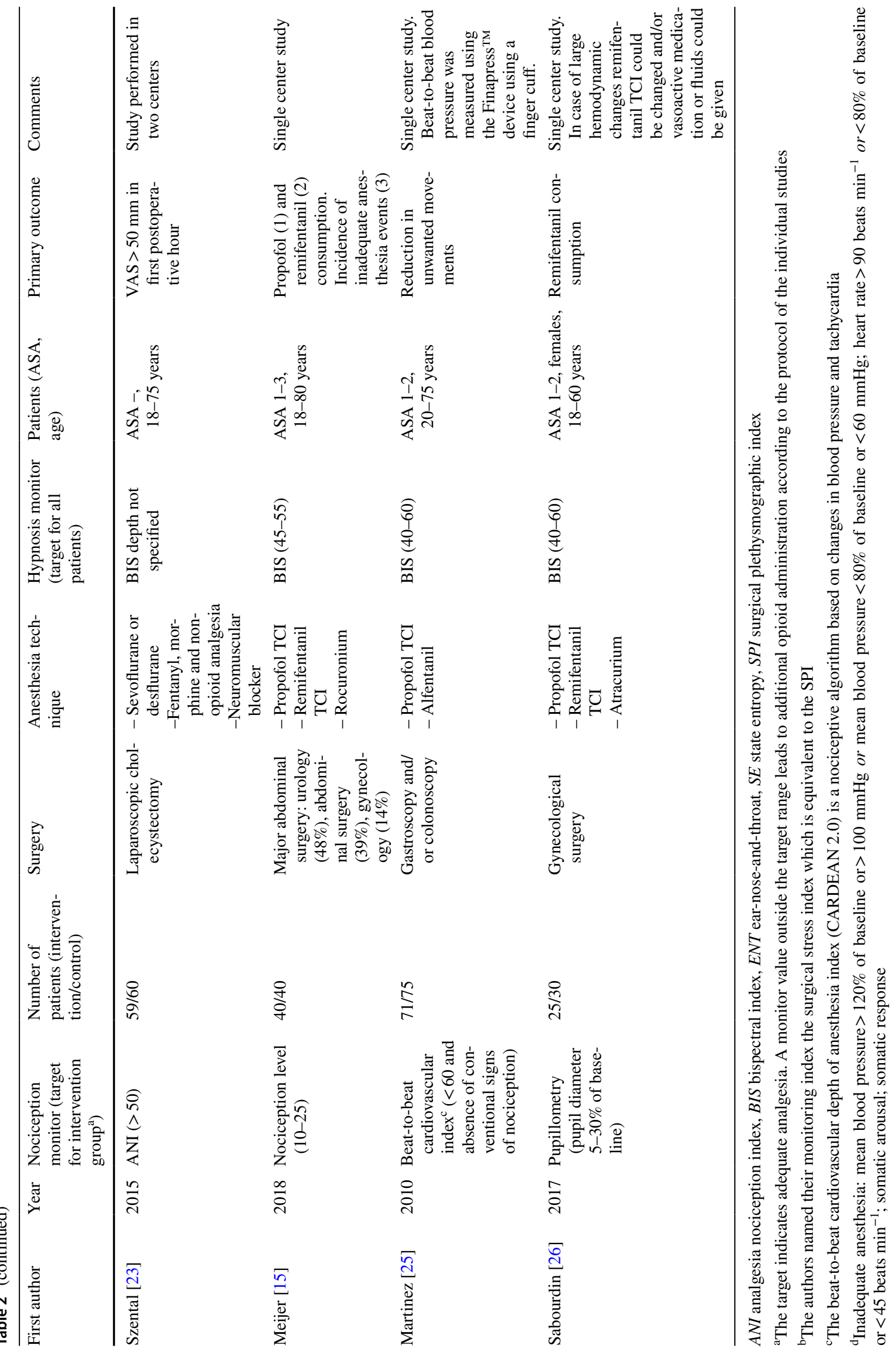




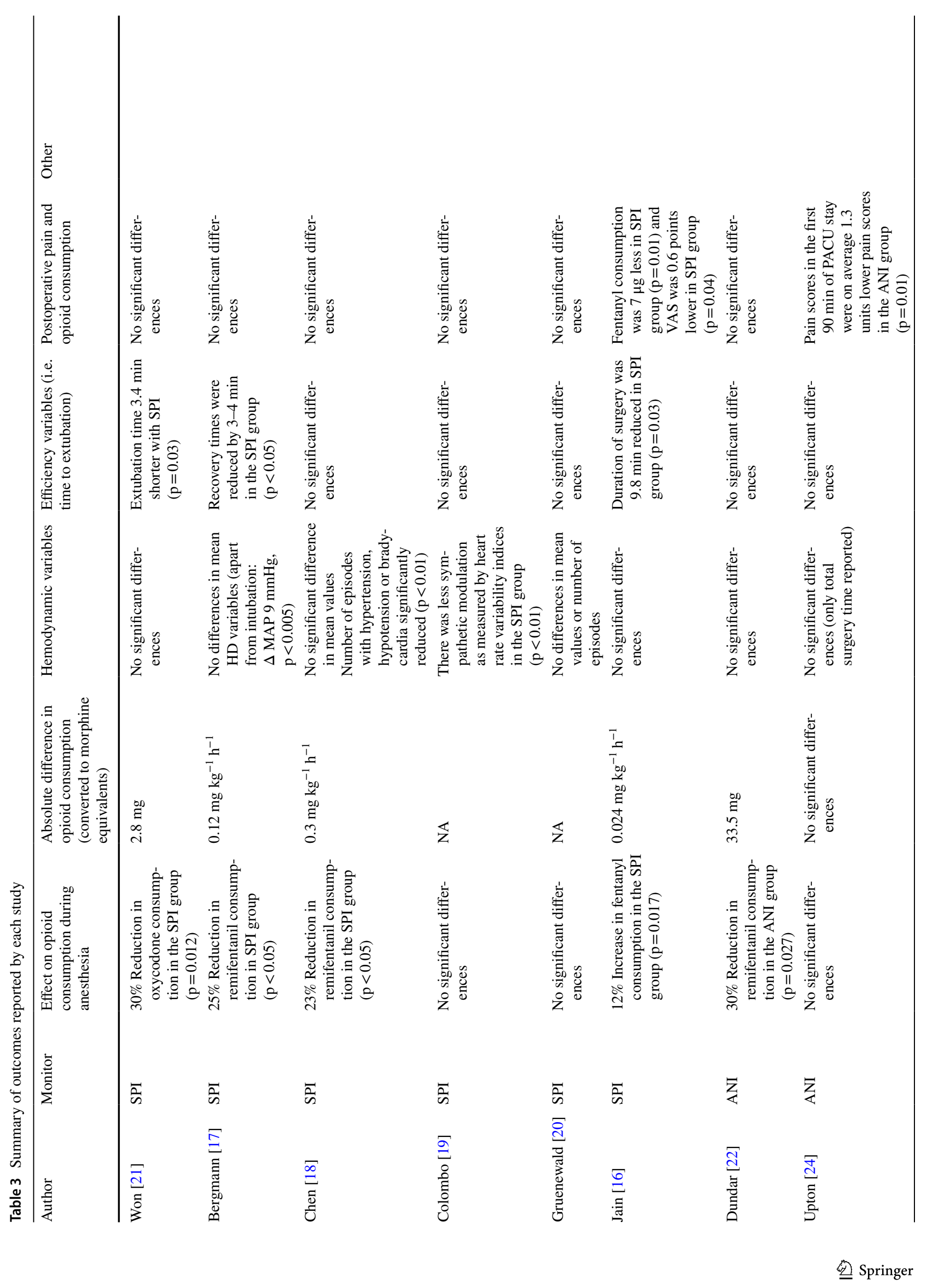




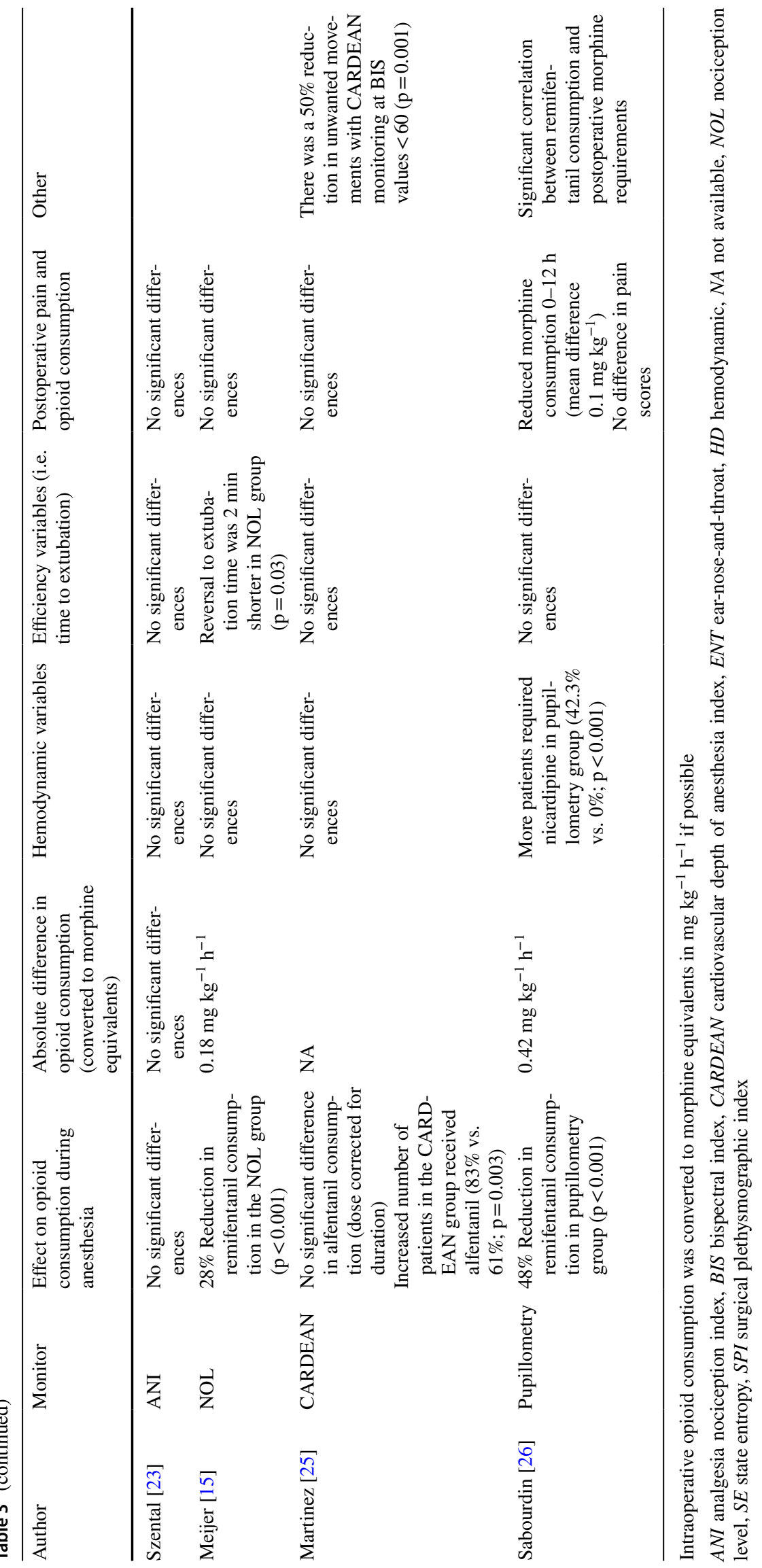


( $30 \%$ or $33.5 \mathrm{mg}$ morphine equivalents in total, $\mathrm{p}=0.027$ ). This significant difference in remifentanil consumption did not translate into faster recovery times or improved pain scores in the PACU. In addition, methodological flaws (for instance, the report fails to detail data collection and blinding procedures) reduce the quality of evidence of this study. The studies of Szental et al. and Upton et al. found no differences in opioid use during anesthesia, however opioid consumption was not the primary outcome of both studies and the use of morphine for intraoperative analgesia may not have been an ideal choice [23, 24]. Regarding other endpoints, only Upton et al., found lower pain scores in ANI guided patients after lumbar discectomy or laminectomy (mean difference first 90 min postoperative 1.3 NRS points, $p=0.01$; see Table 2) [24]. However total fentanyl dose in the PACU was not significantly different.

Pooled data analysis of these studies show that ANI guidance did not result in a significant difference in intraoperative opioid consumption: mean difference $+0.00 \mathrm{mg} \mathrm{kg}^{-1} \mathrm{~h}^{-1}$ morphine ( $95 \% \mathrm{CI}-0.018$ to 0.024 , $\left.\mathrm{Z}=0.12, \mathrm{p}=0.90, \mathrm{I}^{2}=98 \%\right)$.

In conclusion, analysis of the pooled data did not show a benefit of ANI guidance on intraoperative opioid consumption. Preliminary effects of ANI monitoring concerning an opioid sparing effect during breast surgery in patients that receive additional neuraxial blockade or on postoperative pain scores after back surgery, need to be corroborated in future studies.

\subsubsection{Nociception level index (NOL)}

Our systematic review did not find any study that compared NOL guided anesthesia versus standard care on intraoperative opioid consumption. However, our group recently published a trial in which NOL guided anesthesia was compared to standard care in 80 patients during major ambulatory laparoscopic and open abdominal surgery without the use of neuraxial blockade [15]. General anaesthesia was maintained with propofol (bispectral index target 40-60) and remifentanil (NOL target 10-25 for the intervention group; blinded for standard care group). Propofol and remifentanil were administered using target controlled infusion. This study found a reduction in remifentanil consumption of $28 \%$ (absolute reduction $0.18 \mathrm{mg} \mathrm{kg}^{-1} \mathrm{~h}^{-1}$ morphine equivalents; $\mathrm{p}<0.001$ ). Additionally this study found a trend towards improved hemodynamic stability. Postoperative pain scores or opioid consumption did not differ significantly (see Table 2).

In conclusion, data from only one study indicates that NOL guided anesthesia may reduce intraoperative remifentanil consumption. Future studies are needed.

\subsubsection{Cardiovascular depth of analgesia (CARDEAN 2.0)}

The systematic search yielded one study that assessed the use of the CARDEAN 2.0 monitor versus standard care during procedural sedation for endoscopic procedures [27]. Sedation was administered with the use of targetcontrolled infusion propofol, aimed at a BIS of 60. Additionally, in the CARDEAN group, alfentanil could be administered when the monitor value exceeded 60 . In the standard care, alfentanil could only be administered if a mandatory propofol intervention failed to achieve stability. Due to the nature of this protocol, the CARDEAN group received more doses of alfentanil, although the normalized dose (corrected for procedure time) was not significantly different. The increased use of alfentanil in the CARDEAN group resulted in significantly less unwanted movements during the procedures ( $50 \%$ reduction, $\mathrm{p}=0.001$ ), but also a tendency to increased apnea [27].

In conclusion, limited evidence from a single center study shows an increase in opioid administration and a reduction in unwanted movements when the CARDEAN is used for procedural sedation. The possibility that the increase in opioid administration is related to the study protocol itself cannot be ruled out.

\subsubsection{Pupillometry}

Pupillometry versus standard care was assessed in one study in 55 patients during major gynaecological surgery [26]. In both groups, anaesthesia was maintained with propofol, aimed at a BIS of 40-60. In the pupillometry group, remifentanil was dosed according to predefined changes in pupil diameter. This study found reduced remifentanil consumption in pupillometry guided patients (mean difference $0.42 \mathrm{mg} \mathrm{kg}^{-1} \mathrm{~h}^{-1}$ morphine equivalents, $\mathrm{p}<0.001$ ). This translated to less morphine requirement in the first $12 \mathrm{~h}$ after surgery. Pain scores did not differ significantly. In addition, persistent pain was less frequent after 3 months post-surgery in pupillometry guided patients $(51 \%$ in standard group vs. $13 \%$ in pupillometry group, $p=0.004)$. The reduced intraoperative remifentanil administration in pupillometry group resulted in more administration of nicardipine for hypertensive episodes (see Table 2) [26].

In conclusion, data from one study indicates that nociception monitoring by pupillometry may help to reduce opioid consumption during major gynaecological surgery, with possible secondary benefits on short term (less opioid consumption) and midterm (less persistent pain). Future studies are however needed. 


\subsection{Risk of bias}

Risk of bias per study is shown in Fig. 2a and summarized in Fig. 2b. All studies were troubled by the inability to fully blind the investigators due to the nature of the intervention, i.e. need to either use or not-use the monitor during the surgical procedure. Setting aside this inevitable performance risk, seven studies had no additional high risk of bias domain according to the definitions of the Cochrane Handbook for Systematic Reviews of Interventions [15-17, 20,21, 23, 24], two studies had one additional high risk of bias [18, 19]; and the remaining three studies had two or three additional high risks of bias domains $[22,25,26]$. The most common high risk of bias, apart from the performance bias, was detection bias (five studies) $[18,19,22,25,26]$ and other bias (three studies) $[22,25,26]$.

Fig. 2 Evaluation of bias in the selected 12 studies. a Risk of bias per study according to the domains defined in the Cochrane Handbook for Systematic Reviews of Interventions version 5.1.0 (http://handb ook-5-1.cochrane.org). b Summary of bias per domain

\section{Discussion}

Our systematic search on the effect of nociception monitoring versus standard care during general anesthesia on opioid consumption yielded 12 reports. Four did not find a significant difference in opioid consumption, seven found a reduced opioid consumption-with widely varying magnitude and one study found an increased opioid consumption (see Table 3). We did not conduct a planned meta-analysis because the data were so heterogeneous. However, a metaanalysis has been published before by Gruenewald et al., despite substantial heterogeneity. They found no significant effect of nociception monitoring on intraoperative opioid consumption or other secondary outcomes, apart from a reduced rate of unwanted movement [7].

We performed sub-analyses of pooled data from unique monitors when more than one trial was available which was the case for the SPI and ANI monitors. These analyses

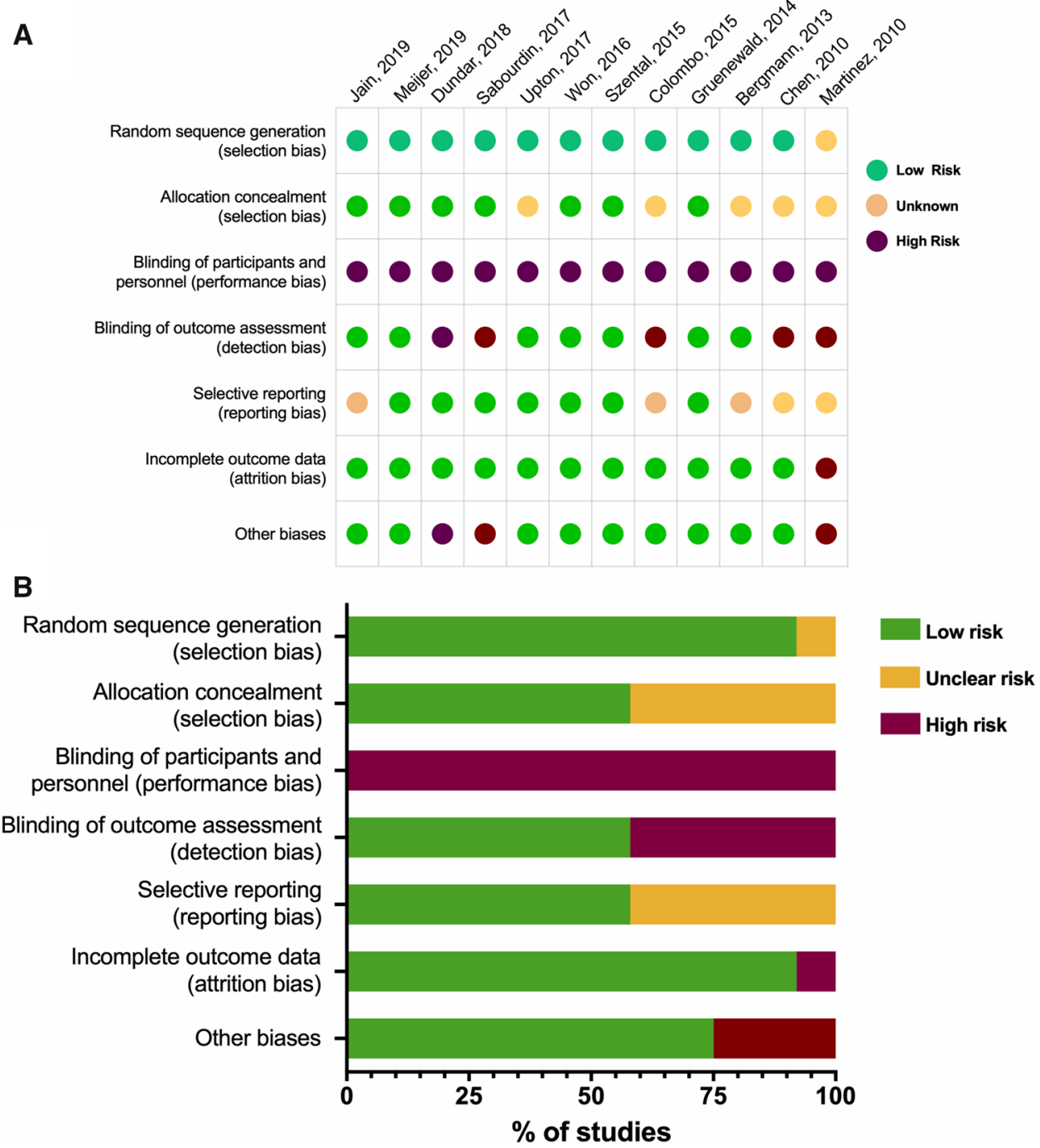


found no repeatable significant effect for the ANI monitor but an opioid sparing effect for the SPI monitor of about $8 \%$ or $0.06 \mathrm{mg} \mathrm{kg}^{-1} \mathrm{~h}^{-1}$ morphine equivalents. It is debatable whether this reduction is clinically relevant. Data from single studies concerning the NOL and pupillometry monitors also show a more substantial reduction in intraoperative opioid consumption ( 0.18 and $0.42 \mathrm{mg} \mathrm{kg}^{-1} \mathrm{~h}^{-1}$ morphine equivalents respectively) $[15,26]$. However, these results require confirmation. On secondary outcomes, no consistent beneficial effect of nociception guided management was observed, although admittedly, some individual well conducted trials show promising effects on intraoperative hemodynamic stability [15] or postoperative pain [16, 24 , 26] (see Table 2). Again, these results need to be confirmed.

Measurement of nociception under general anesthesia is challenging task. In general, noxious stimuli that are perceived by the autonomic nerve system will evoke autonomic and behavioral responses. The magnitude of these responses depends on the intensity of the stimulus and the presence of any alleviating agents; i.e. the nociception-anti-nociception balance. During anesthesia, autonomic responses are noted by their effects on haemodynamic and respiratory control. Nociception monitors invariably use one or more of these autonomic variables as input for their algorithm to produce an index of nociception (see Table 3). Unfortunately, these autonomic variables are not uniquely related to nociception; any stressing or alleviating factor may cause a change in blood pressure or heart rate. In addition, both the choice of maintenance hypnotic and opioid and the type of surgical procedure profoundly affect the nociception-anti-nociception balance. All these factors may reduce the specificity of nociception monitors when they are tested in the clinical setting.

Patients show large inter-individual differences in their response to noxious stimuli and analgesic therapy. Nociception monitors can be used as an aid to improve individualized antinociceptive therapy in daily practice, however they should not be used to maintain nociception index values within a specific range at all costs. In addition, although some trials showed a reduced opioid consumption in nociception guided patients, the use of these monitors can also result in an increased consumption of opioids or other analgesics for certain procedures [16]. Both ways may improve outcomes for patients, such as reduced postoperative pain. The key utility of these monitors is not reducing opioid consumption per se, rather to achieve the optimal dosing of any analgesic technique for the individual patient that will result in the best outcome.

\subsection{General limitations}

Current data are troubled by the large heterogeneity that was present among studies. Studies differed significantly in design, study population and surgery and anesthesia type (Table 1). The most commonly performed type of surgery was abdominal surgery (five studies, some of which were laparoscopic) $[15,16,19,20,23,26]$; other procedures included ear-nose-and-throat surgery [18], breast surgery [22], thyroidectomy [21], lumbar discectomy/laminectomy [24], and orthopedic surgery (arthroscopy of knee, shoulder or ankle) [17]. Anesthesia technique varied considerably among studies: six studies used propofol for maintenance of anesthesia [15, 17-19, 25, 26], five combined with remifentanil and one combined with alfentanil. The other studies used a volatile anesthetic (sevoflurane or desflurane) combined with either remifentanil $(n=1)$, fentanyl $(n=2)$, oxycodone $(n=1)$ or sufentanil $(n=1)$ [16, 20-24]. Most studies used a neuromuscular blocker $(n=9)[15-21,23$, $24,26]$, and one study used a thoracic paravertebral block in both intervention and standard care groups [22]. The ample differences in type of anesthesia and in the intensity of the surgical trauma may profoundly affect the individual study results. For instance, noxious stimuli during gastroscopy under propofol/alfentanil anesthesia will differ significantly in intensity from stimuli during discectomy under sevoflurane/fentanyl anesthesia or breast surgery under sevoflurane/ fentanyl surgery with a thoracic paravertebral local anesthetic block [22, 24].

Also, the comparator arm in most trials (commonly defined as standard clinical care) often lacked strict guidelines for opioid administration. Therefore, any effect of nociception monitoring on opioid administration could be confounded by suboptimal clinical practice of the comparator group. Finally, all studies included in our review were relatively small with fewer than 100 subjects per treatment arm, and most had fewer than 50 per arm. Additionally, all studies had a high risk of performance bias and five studies had a high risk of detection bias (Fig. 2).

\subsection{Limitations of this review}

Although our search was extensive, it was limited to the PubMed database and we may have missed some relevant studies. We therefore performed a secondary search across websites of anesthesia societies to detect studies that are presented as abstracts. One possible relevant abstract was detected. Gruenewald et al. performed a multi-center, single blinded randomized-controlled trial in 494 patients studying the influence of SPI and entropy monitoring (i.e. combined analgesia and hypnosis monitoring) versus standard monitoring on signs of unwanted anesthesia events [28]. However, in their preliminary report the authors do not mention opioid consumption, which is the endpoint of this review. No other potentially relevant reports were found. Finally, we only included studies on adult patients, and we did not report on nociception monitoring in children. 


\section{Conclusions}

Current data are inconclusive about the effect of nociception monitoring on intraoperative opioid consumption or anesthesia-related outcome. Future homogeneous (randomized and open) and predefined (to reduce heterogeneity and detection risk) trials are needed to improve current level of evidence.

Funding This study was supported by institutional and departmental funds.

\section{Compliance with ethical standards}

Conflict of interest AD received Speaker Fee from Medasense Biometrics Ltd. (Israel); RE and DIS serve on an Advisory Board for Medasense Biometrics Ltd. and have an Equity Interest in the company. Medasense is the Developer of the nociception level index. The other authors declare no competing interests.

Open Access This article is distributed under the terms of the Creative Commons Attribution 4.0 International License (http://creativeco mmons.org/licenses/by/4.0/), which permits unrestricted use, distribution, and reproduction in any medium, provided you give appropriate credit to the original author(s) and the source, provide a link to the Creative Commons license, and indicate if changes were made.

\section{References}

1. Cantineau JP, Porte F, d'Honneur G, Duvaldestin P. Neuromuscular effects of rocuronium on the diaphragm and adductor pollicis muscles in anesthetized patients. Anesthesiology. 1994;81(3):585-90.

2. Edry R, Recea V, Dikust Y, Sessler DI. Preliminary intraoperative validation of the nociception level index: a noninvasive nociception monitor. Anesthesiology. 2016;125(1):193-203. https://doi. org/10.1097/aln.0000000000001130.

3. Funcke S, Sauerlaender S, Pinnschmidt HO, Saugel B, Bremer K, Reuter DA, Nitzschke R. Validation of innovative techniques for monitoring nociception during general anesthesia: a clinical study using tetanic and intracutaneous electrical stimulation. Anesthesiology. 2017;127(2):272-83. https://doi.org/10.1097/aln.00000 00000001670

4. Ledowski T, Burke J, Hruby J. Surgical pleth index: prediction of postoperative pain and influence of arousal. Br J Anaesth. 2016;117(3):371-4. https://doi.org/10.1093/bja/aew226.

5. Stockle PA, Julien M, Issa R, Decary E, Brulotte V, Drolet P, Henri M, Poirier M, Latulippe JF, Dorais M, Verdonck O, Fortier LP, Richebe P. Validation of the PMD100 and its NOL Index to detect nociception at different infusion regimen of remifentanil in patients under general anesthesia. Minerva Anestesiol. 2018;84(10):1160-8. https://doi.org/10.23736/s0375 -9393.18.12720-9.

6. Martini CH, Boon M, Broens SJ, Hekkelman EF, Oudhoff LA, Buddeke AW, Dahan A. Ability of the nociception level, a multiparameter composite of autonomic signals, to detect noxious stimuli during propofol-remifentanil anesthesia. Anesthesiology. 2015;123(3):524-34. https://doi.org/10.1097/aln.0000000000 000757.
7. Gruenewald M, Dempfle A. Analgesia/nociception monitoring for opioid guidance: meta-analysis of randomized clinical trials. Minerva Anestesiol. 2017;83(2):200-13. https://doi.org/10.23736 /s0375-9393.16.11602-5.

8. Knotkova H, Fine PG, Portenoy RK. Opioid rotation: the science and the limitations of the equianalgesic dose table. J Pain Symptom Manag. 2009;38(3):426-39. https://doi.org/10.1016/j.jpain symman.2009.06.001.

9. Higgins JP, Altman DG, Gotzsche PC, Juni P, Moher D, Oxman AD, Savovic J, Schulz KF, Weeks L, Sterne JA, Cochrane Bias Methods Group, Cochrane Statistical Methods Group. The Cochrane Collaboration's tool for assessing risk of bias in randomised trials. BMJ (Clin Res Ed.). 2011;343:d5928. https://doi. org/10.1136/bmj.d5928.

10. R Core Team. R: a language and environment for statistical computing. Vienna: R Foundation for Statistical Computing; 2018. https://www.R-project.org/. Accessed 22 July 2018.

11. Viechtbauer W. Conducting meta-analyses in $\mathrm{R}$ with the metafor package. 2010. https://www.jstatsoft.org/article/view/. Accessed 22 July 2018.

12. Defresne A, Barvais L, Clement F, Bonhomme V. Standardised noxious stimulation-guided individual adjustment of remifentanil target-controlled infusion to prevent haemodynamic responses to laryngoscopy and surgical incision: a randomised controlled trial. Eur J Anaesthesiol. 2018;35(3):173-83. https://doi.org/10.1097/ eja.0000000000000742.

13. Le Gall L, David A, Carles P, Leuillet S, Chastel B, Fleureau C, Dewitte A, Ouattara A. Benefits of intraoperative analgesia guided by the Analgesia Nociception Index (ANI) in bariatric surgery: an unmatched case-control study. Anaesth Crit Care Pain Med. 2019;38(1):35-9. https://doi.org/10.1016/j.accpm.2017.09.004.

14. Rogobete AF, Sandesc D, Cradigati CA, Sarandan M, Papurica M, Popovici SE, Vernic C, Bedreag OH. Implications of Entropy and Surgical Pleth Index-guided general anaesthesia on clinical outcomes in critically ill polytrauma patients. A prospective observational non-randomized single centre study. J Clin Monit Comput. 2018;32(4):771-8. https://doi.org/10.1007/s10877-017-0059-2.

15. Meijer F, Martini C, Broens S, Boon M, Niesters M, Aarts L, Olofsen E, van Velzen M, Dahan A. Nociception-guided versus standard care during remifentanil-propofol anesthesia: a randomized controlled trial. Anesthesiology 2019;130(5):745-5. https ://doi.org/10.1097/ALN.0000000000002634

16. Jain N, Gera A, Sharma B, Sood J, Chugh P. Comparison of surgical pleth index guided analgesia using fentanyl versus conventional analgesia technique in laparoscopic cholecystectomy. Minerva Anestesiol. 2019. https://doi.org/10.23736/s0375 -9393.18.12954-3.

17. Bergmann I, Gohner A, Crozier TA, Hesjedal B, Wiese CH, Popov AF, Bauer M, Hinz JM. Surgical pleth index-guided remifentanil administration reduces remifentanil and propofol consumption and shortens recovery times in outpatient anaesthesia. Br J Anaesth. 2013;110(4):622-8. https://doi.org/10.1093/bja/aes426.

18. Chen X, Thee C, Gruenewald M, Wnent J, Illies C, Hoecker J, Hanss R, Steinfath M, Bein B. Comparison of surgical stress index-guided analgesia with standard clinical practice during routine general anesthesia: a pilot study. Anesthesiology. 2010;112(5):1175-83. https://doi.org/10.1097/aln.0b013e3181 d3d641.

19. Colombo R, Raimondi F, Rech R, Castelli A, Fossali T, Marchi A, Borghi B, Corona A, Guzzetti S. Surgical Pleth Index guided analgesia blunts the intraoperative sympathetic response to laparoscopic cholecystectomy. Minerva Anestesiol. 2015;81(8):837-45.

20. Gruenewald M, Willms S, Broch O, Kott M, Steinfath M, Bein B. Sufentanil administration guided by surgical pleth index vs standard practice during sevoflurane anaesthesia: a randomized 
controlled pilot study. Br J Anaesth. 2014;112(5):898-905. https ://doi.org/10.1093/bja/aet485.

21. Won YJ, Lim BG, Lee SH, Park S, Kim H, Lee IO, Kong MH. Comparison of relative oxycodone consumption in surgical pleth index-guided analgesia versus conventional analgesia during sevoflurane anesthesia: a randomized controlled trial. Medicine (Baltim.). 2016;95(35):e4743. https://doi.org/10.1097/md.00000 00000004743.

22. Dundar N, Kus A, Gurkan Y, Toker K, Solak M. Analgesia nociception index (ANI) monitoring in patients with thoracic paravertebral block: a randomized controlled study. J Clin Monit Comput. 2018;32(3):481-6. https://doi.org/10.1007/s10877-017-0036-9.

23. Szental JA, Webb A, Weeraratne C, Campbell A, Sivakumar H, Leong S. Postoperative pain after laparoscopic cholecystectomy is not reduced by intraoperative analgesia guided by analgesia nociception index (ANI(R)) monitoring: a randomized clinical trial. Br J Anaesth. 2015;114(4):640-5. https://doi.org/10.1093/ bja/aeu411.

24. Upton HD, Ludbrook GL, Wing A, Sleigh JW. Intraoperative "Analgesia Nociception Index"-guided fentanyl administration during sevoflurane anesthesia in lumbar discectomy and laminectomy: a randomized clinical trial. Anesth Analg. 2017;125(1):8190. https://doi.org/10.1213/ane.0000000000001984.

25. Martinez JY, Wey PF, Lions C, Cividjian A, Rabilloud M, Bissery A, Bourdon L, Puidupin M, Escarment J, Quintin L. A beat-bybeat cardiovascular index, CARDEAN: a prospective randomized assessment of its utility for the reduction of movement during colonoscopy. Anesth Analg. 2010;110(3):765-72. https://doi. org/10.1213/ane.0b013e3181cc9ebe.

26. Sabourdin N, Barrois J, Louvet N, Rigouzzo A, Guye ML, Dadure $\mathrm{C}$, Constant I. Pupillometry-guided intraoperative remifentanil administration versus standard practice influences opioid use: a randomized study. Anesthesiology. 2017;127(2):284-92. https:// doi.org/10.1097/aln.0000000000001705.

27. Rossi M, Cividjian A, Fevre MC, Oddoux ME, Carcey J, Halle C, Frost M, Gardellin M, Payen JF, Quintin L. A beat-by-beat, on-line, cardiovascular index, CARDEAN, to assess circulatory responses to surgery: a randomized clinical trial during spine surgery. J Clin Monit Comput. 2012;26(6):441-9. https://doi. org/10.1007/s10877-012-9372-y.

28. Grüenewald M, Harjuz J, Preckel B, Moinár Z, Yli-Hankalas A, Beins B. Comparison of adequacy of anaesthesia monitoring with standard clinical practice during routine general anaesthesia: an international, multi-centre, single-blinded randomized controlled trial. In: Abstract 1718. Paper presented at the Euroanaesthesia, Copenhagen, 3 June 2018.

Publisher's Note Springer Nature remains neutral with regard to jurisdictional claims in published maps and institutional affiliations. 